Pacific Journal of Mathematics

A TOPOLOGICAL LEMMA AND APPLICATIONS TO REAL 


\title{
A TOPOLOGICAL LEMMA AND APPLICATIONS TO REAL FUNCTIONS
}

\author{
ClifFord E. WeIL
}

\begin{abstract}
In working with functions of Baire class one having the Darboux property, one of the most useful tools has been a theorem due to Baire that says a function of Baire class one has a point of continuity on every closed set relative to the closed set. The lemma mentioned in the title can be used in many instances more efficiently than Baire's theorem as is shown in $\S 4$. It is concerned with sets rather than functions and hence more basic than Baire's Theorem, and easier to prove requiring only one application of Baire's category theorem.
\end{abstract}

1. Definitions and conventions. Euclidean $n$-space will be denoted by $E_{n}$ and $K$ will designate a fixed, closed subset of $E_{n}$. For each $x$ in $K$ and $r$ positive, $B(x, r)=\{y \in K:|x-y|<r\}$; that is, the open ball of radius $r$ about $x$. For any subset $H$ of $K$, cl $H$ will be its closure and int $H$ will denote its interior relative to $K$. Finally $m(E)$ will be the Lebesgue measure of $E$.

Definition 1. A $G_{\delta}$ subset $H$ of $K$ will be called a ball closed $G_{\delta}$ set if whenever $B(x, r) \subset H,\{y \in K:|x-y|=r\} \subset H$.

Clearly every closed set is a ball closed $G_{\delta}$ set. However, not every ball closed $G_{\delta}$ set is closed. For example, in $[0,1]$ the set

$$
H=\bigcup_{n=2}^{\infty}\left[n^{-1}, n^{-1}+2^{-n}\right]
$$

is a ball closed $G_{\delta}$ set that is not closed because it does not contain 0 . Not every $G_{\delta}$ set is a ball closed $G_{\delta}$ set. In particular it is fairly easy to prove (using one of the techniques used in the proof of the lemma) that the only nonempty open subset of $K$ that is a ball closed $G_{\delta}$ set is $K$ itself. This fact will be needed.

Definition 2. A function $f: E_{n} \rightarrow E_{k}$ has the Denjoy property if for each open subset $G$ of $E_{k}$ either $f^{-1}(G)=\varnothing$ or $m\left(f^{-1}(G)\right)>0$.

\section{The lemma.}

LEMMA. If $H_{1}$ and $H_{2}$ are two nonempty disjoint, ball closed $G_{\delta}$ subsets of $K$, then there is an $x \in K-\left(H_{1} \cup H_{2}\right)$, and an open neighborhood $O$ of $x$ (open relative to $K$ ) such that $O \cap H_{1}=\varnothing$ or $O \cap H_{2}=\varnothing$. 
Proof. Suppose, on the contrary, that every open neighborhood of every point $x \in K-\left(H_{1} \cup H_{2}\right)$ intersects both $H_{1}$ and $H_{2}$. The first step of the proof is establishing the following fact. Every open set intersecting $H_{1}$-int $H_{1}$ also intersects $H_{2}$-int $H_{2}$, and conversely, every open set intersecting $H_{2}$ - int $H_{2}$ also intersects $H_{1}-\operatorname{int} H_{1}$. It will suffice to prove the first part; the proof of the second being similar.

Let $x \in H_{1}-\operatorname{int} H_{1}, U$ an open set with $x \in U$ and $r>0$ so that $B(x, r) \subset U$. Since $x \notin$ int $H_{1}$, there is a $y \in K-H_{1}$ with $|x-y|<r / 2$. Either $y \in H_{2}$ or $y \in K-H_{2}$ in which case $y \in K-\left(H_{1} \cup H_{2}\right)$; so since $B(x, r / 2)$ is an open set containing $y$, by assumption $B(x, r / 2)$ contains a point of $H_{2}$. In either case there is a point $z \in B(x, r / 2) \cap H_{2}$. If $z \notin$ int $\mathrm{H}_{2}$ it is the desired point. If not to find the desired point let

$$
s=\sup \left\{t: B(z, t) \subset H_{2}\right\} .
$$

Since $x \in H_{1}$ and $H_{1} \cap H_{2}=\varnothing, s \leqq r / 2$ (actually $s<r / 2$, but that fact is not needed). A simple compactness argument then shows that there is a point $w$ with $|z-w|=s$ such that $w \in K-\operatorname{int} H_{2}$. But since $H_{2}$ is a ball closed $G_{\delta}$ set, $w \in H_{2}$. Moreover,

$$
|x-w| \leqq|x-z|+|z-w|<r / 2+s \leqq r .
$$

Hence

$$
w \in U \cap\left(H_{2}-\operatorname{int} H_{2}\right) \text {. }
$$

Let

$$
F=\operatorname{cl}\left[\left(H_{1}-\operatorname{int} H_{1}\right) \cup\left(H_{2}-\operatorname{int} H_{2}\right)\right] \text {. }
$$

The fact just verified says that both $H_{1}$ and $H_{2}$ are dense in $F$. Now $H_{1}$ and $H_{2}$ are $G_{\delta}$ sets; so write

$$
H_{1}=\bigcap_{n=1}^{\infty} U_{n}
$$

and

$$
H_{2}=\bigcap_{n=1}^{\infty} V_{n}
$$

where $U_{n}$ and $V_{n}$ are open sets (relative to $K$ ) for each $n=1,2, \cdots$. Then for each $n=1,2, \cdots, U_{n} \cap F$ and $V_{n} \cap F$ are open subsets of $F$ and each contains a dense subset of $F$. The intersection of two such sets is a dense set; so $U_{n} \cap V_{n} \cap F$ is an open, dense subset of $F$. So by the Baire category theorem,

$$
\bigcap_{n=1}^{\infty}\left(U_{n} \cap V_{n} \cap F\right)
$$


is a dense subset of $F$. But

$$
\begin{aligned}
\bigcap_{n=1}^{\infty}\left(U_{n} \cap V_{n} \cap F\right) & =\left(\bigcap_{n=1}^{\infty} U_{n}\right) \cap\left(\bigcap_{n=1}^{\infty} V_{n}\right) \cap F \\
& =H_{1} \cap H_{2} \cap F=\varnothing .
\end{aligned}
$$

So $F=\varnothing$; that is $H_{1}-\operatorname{int} H_{1}=\varnothing$ and $H_{2}-\operatorname{int} H_{2}=\varnothing$. That is, $H_{1}$ and $H_{2}$ are both open, ball closed $G_{\delta}$ sets which are nonempty. By the remark following the definition of ball closed $G_{\delta}$ sets, $H_{1}=$ $K=H_{2}$ contrary to the assumption that they are disjoint.

One immediate consequence of the lemma that will be used in the next section and in $\S 6$ is that a closed subset of $K$ cannot be decomposed into two, nonempty disjoint, ball closed $G_{\delta}$ sets.

3. Applications to Baire one, Darboux functions, A function $f: E_{1} \rightarrow E_{1}$ of Baire class one having the Darboux property has a connected graph (see [1] Theorem 4.1). An easy proof of this fact can be given by first observing that if $G$ is an open subset of $E_{2}$, then $\{x:(x, f(x)) \in G\}$ is an $F_{\sigma}$ set. If the graph of $f$ is disconnected by two open sets $O_{1}$ and $O_{2}$, then the two sets $E_{i}=\left\{x:(x, f(x)) \in O_{i}\right\}$, $i=1,2$, are disjoint $F_{\sigma}$ subsets of $E_{1}$ whose union is $E_{1}$. Hence they are also $G_{\delta}$ sets. That $f$ has the Darboux property implies that $E_{1}$ and $E_{2}$ are ball closed $G_{\delta}$ sets. By the lemma either $E_{1}=\varnothing$ or $E_{2}=\varnothing$. A similar argument can be used to give a short proof of Zahorski's classification of Baire one, Darboux functions (see [1] or [10]).

In [6] (also [1] Theorem 6.1) it is shown that a function $f: E_{1} \rightarrow E_{1}$ is of Baire class one and has the Darboux property if and only if for each real number $a$, the sets

$$
E_{a}=\{x: f(x) \geqq a\}
$$

and

$$
E^{a}=\{x: f(x) \leqq a\}
$$

are ball closed $G_{\delta}$ subsets of $E_{1}$. The "if" part follows at once from the remark after the lemma, for if on a closed interval $I, f$ attains values larger than $a$ and smaller than $a$, but does not attain the value $a$, then $E_{a}$ and $E^{a}$ would be a decomposition of $I$ into two nonempty disjoint ball closed $G_{\delta}$ sets.

The following corollary was certainly attainable using Baire's Theorem, but seems to have escaped detection. It will be the crux of many of the remaining applications. 
having the Darboux property, then for each open interval $(a, b)$ with $f^{-1}(a, b) \neq \varnothing$, there is an interval $I$ such that $f^{-1}(a, b) \cap I \neq \varnothing$ and on $I, f$ is strictly larger than a or strictly smaller than $b$.

Proof. By the above result of Neugebauer's the two sets $E_{b}$ and $E^{a}$ are ball closed $G_{\delta}$ sets, and it may be assumed that they are nonempty for otherwise the conclusion is immediate. The lemma then says that there is an interval $I$ and an $x \in I$ such that $x \notin E_{b} \cup E^{a}$ (that is $x \in f^{-1}(a, b)$ ) and $I \cap E_{b}=\varnothing$ or $I \cap E^{a}=\varnothing$ which is the desired result.

4. Applications to derivatives. The proceeding corollary gives an easy proof of the following theorem of Denjoy's (see [2]).

THEOREM. If $f: E_{1} \rightarrow E_{1}$ is everywhere differentiable, then $f^{\prime}$ has the Denjoy property.

Proof. The function $f^{\prime}$ is of Baire class one and has the Darboux property; so if $f^{\prime-1}(a, b) \neq \varnothing$ then by the corollary there is an interval $I$ such that $f^{\prime-1}(a, b) \cap I \neq \varnothing$ and on $I, f^{\prime}$ is strictly larger than $a$ or strictly smaller than $b$.

Thus the theorem will follow by proving the special case that if $f^{\prime}(x)>0$ for all $x \in I$ and $f^{\prime}(x) \geqq 1$ for a.e. $x \in I$, then $f^{\prime}(x) \geqq 1$ for every $x \in I$. This can be established in two ways. Using the Lebesgue theory it follows that $f^{\prime}$ is integrable and for every

$$
x<y, x, y \in I, f(y)-f(x) \geqq \int_{x}^{y} f^{\prime}(t) d t \geqq y-x ;
$$

that is $(f(y)-f(x)) /(y-x) \geqq 1$. Letting $y \rightarrow x$ yields $f^{\prime}(x) \geqq 1$ for every $x \in I$. Using more primitive methods a standard nested interval argument will prove that if the lower Dini derivate of a function is $\geqq 1$ for each $x \in I$, then each difference quotient of the function is $\geqq 1$. Next let $E=\left\{x \in I: f^{\prime}(x)<1\right\}$ and for each $n=1,2, \cdots$, let $G_{n}$ be an open set, $G_{n} \supset E$, with $m\left(G_{n}\right)<1 / n$. Define

$$
f_{n}(x)=m\left((-\infty, x) \cap G_{n}\right)+f(x) .
$$

It is easy to show that the lower Dini derivate of $f_{n}$ is $\geqq 1$ for each $x$ in $I$ and hence that for each $x, y \in I, x \neq y$,

$$
\left(f_{n}(y)-f_{n}(x)\right) /(y-x) \geqq 1 .
$$

But clearly $\left\{f_{n}\right\}$ converges pointwise to $f$. Thus

$$
(f(y)-f(x)) /(y-x) \geqq 1 \text {. }
$$


And as before it follows that $f^{\prime}(x) \geqq 1$ for each $x \in I$.

The Denjoy property is possessed by $L_{p}$-derivatives, approximate derivatives and $k$ th Peano derivatives. For definitions and proofs [3] and [7]. The properties that all of these generalized derivatives have which yield an immediate proof of the result is that they are Baire one functions, have the Darboux property, and if bounded above or below on an interval they are ordinary derivatives on that interval (see [3], [4], and [7]). So by the corollary if one of the generalized derivatives inverses an open interval to a nonempty set $E$, then there is an $x \in E$ and an interval $I$ containing $x$ such that the generalized derivative is bounded above or below on $I$ and hence is an ordinary derivative on $I$. So by Denjoy's theorem

$$
m(E \cap I)>0 \text {. }
$$

Actually a property slightly stronger than the Denjoy property was proved here. Even though the set where the derivative is not an ordinary derivative may have positive measure, it does not contain the inverse image of any open interval. It was shown that if the inverse image of an open interval is not empty, then it meets an interval on which the generalized derivative is an ordinary derivative. (This fact was first proved for approximate derivatives by Mukhopadhyay. He gave a standard proof using Baire's theorem). In particular, the generalized derivative, when restricted to the set where it is an ordinary derivative, has the Denjoy property. For standard proofs of this fact see [3] and [9].

5. An application to functions of several variables. In [5] Mišik introduced a Darboux property for functions of several variables.

Definition 3. A function $f: E_{n} \rightarrow E_{1}$ has the Darboux property if whenever it attains a value larger than $a$ and a value smaller than $a$ on a closed ball $\left\{y \in E_{n}:|x-y| \leqq r\right\}$, then it attains $a$ on the open ball $\left\{y \in E_{n}:|x-y|<r\right\}$.

The lemma will now be used to prove Mišik's theorem concerning this Darboux property.

THEOREM. A function $f: E_{n} \rightarrow E_{1}$ of Baire class one has the Darboux property if and only if for each real number a, the two sets

$$
E_{a}=\{x: f(x) \geqq a\}
$$

and 


$$
E^{a}=\{x: f(x) \leqq a\}
$$

are ball closed $G_{\delta}$ subsets of $E_{n}$.

Proof. The "only if" part is straightforward and does not need the lemma.

For the "if" part, let $B$ be a closed ball on which $f$ attains a value larger than $a$ and one smaller than $a$. Then $f$ must attain a value larger than $a$ on the interior of $B$, for if not the interior of $B$ would be part of $E^{a}$ and then by hypothesis so would $B$ contrary to assumptions on $B$. Likewise $f$ attains values larger than $a$ on $B$. Let $K$ be a closed ball contained in the interior of $B$, on which $f$ attains values larger than $a$ and smaller. Then $K \cap E_{a}$ and $K \cap E^{a}$ are nonempty ball closed $G_{\delta}$ subsets of $K$ and their union is $K$. But a closed subset of $E_{n}$ cannot be decomposed into nonempty, disjoint, ball closed $G_{\delta}$ subsets. Thus $\left(K \cap E_{a}\right) \cap\left(K \cap E^{a}\right) \neq \varnothing$. If $x$ is in this intersection, then $x$ is interior to $B$ because $K$ is and $f(x)=a$ because $x \in E_{a} \cap E^{a}$.

6. The Darboux property and partial derivatives. This work is concluded by an example, a theorem and some remarks concerning how Mišik's Darboux property relates to partial derivatives. It is shown that a partial derivative even of a continuous function need not have the property, but if the function is differentiable then the partial derivatives, (in fact, all directional derivatives) do satisfy the condition.

Definition 4. If $\nu \in E_{n},|\nu|=1, f: E_{n} \rightarrow E_{1}, x \in E_{n}$, then the directional derivative of $f$ at $x$ in the direction $\nu$ is denoted by $\partial_{\nu} f(x)$ and defined by

$$
\partial_{\nu} f(x)=\lim _{h \rightarrow 0}(f(x+h \nu)-f(x)) / h .
$$

(In $E_{2}$ this notation is a bit clumsy; so there $\partial_{x} f$ and $\partial_{y} f$ are used to denote the usual partial derivatives.)

DEFINITION 5. Let $f: E_{n} \rightarrow E_{1}$, let $\nu^{1}, \cdots, \nu^{n}$ denote the usual basis of unit vectors in $E_{n}$, and let $x \in E_{n}$. Then the gradient of $f$ at $x$ is denoted by grad $f(x)$ and is the vector in $E_{n}$ whose kth coordinate is $\partial_{\nu k} f(x)$.

Definition 6. Let $F: E_{n} \rightarrow E_{1}$ and let $x \in E_{n}$. Then $f$ is said to be differentiable at $x$ if $f(y)=f(x)+(y-x) \cdot \operatorname{grad} f(x)+o(|y-x|)$ as $y \rightarrow x$. 
EXAMPLE. Let $h$ be a differentiable function of one variable that is 0 on $(-\infty,-2]$, constant on $[2, \infty)$ and has derivative 1 on $[-1,1]$. Define $f: E_{2} \rightarrow E_{1}$ by

$$
f(x, y)=\left\{\begin{array}{ll}
\sqrt{y} h(x / \sqrt{y}) & \text { if } y>0 \\
0 & \text { if } y \leqq 0
\end{array} .\right.
$$

Since $h$ is bounded, $f$ is continuous at each point $(x, 0)$. The continuity elsewhere is immediate. It is easily shown that

$$
\partial_{x} f(x, y)= \begin{cases}h^{\prime}(x / \sqrt{y}) & \text { if } y>0 \\ 0 & \text { if } y \leqq 0\end{cases}
$$

In particular $\partial_{x} f(x, y)=1$ if $x^{2} \leqq y$ and $y>0$, but $\partial_{x}(0,0)=0$. It follows then that $E=\left\{(x, y): \partial_{x} f(x, y) \geqq 1\right\}$ contains the open ball $B((0, r), r)$ for any $0<r<1 / 2$, but $(0,0)$, which is on the boundary of such a ball, is not in $E$. Thus $E$ is not a ball closed $G_{\delta}$ set.

TheoRem. If $f$ is differentiable, $\nu \in E_{n}|\nu|=1, a \in E_{1}$, then the sets $E_{\nu}^{a}=\left\{x: \partial_{\nu} f(x) \leqq a\right\}$ and $E_{\nu, a}=\left\{x: \partial_{\nu} f(x) \geqq a\right\}$ are ball closed $G_{\delta}$ sets.

Proof. It suffices to prove that $E_{\nu, 0}$ is a ball closed $G_{\delta}$ set. Let $B(y, r) \subset E_{\nu, 0}$ and let $x$ be such that $|y-x|=r$. For $0<s<1$ the two points

$$
x+s(y-x)=x_{1}
$$

and

$$
x+s(y-x)+\operatorname{sr\nu } / 2=x_{2}
$$

both lie in $B(y, r)$ since

$$
\left|x_{1}-y\right|=(1-s)|y-x|<|y-x|=r
$$

and

$$
\left|x_{2}-y\right| \leqq(1-s)|y-x|+r s / 2=(1-s / 2) r<r .
$$

The line segment joining $x_{1}$ and $x_{2}$ lies entirely in $B(y, r)$. So by the Mean Value Theorem for each $0<s<1$, there is a $0<t<s$ with

$$
f\left(x_{2}\right)-f\left(x_{1}\right)=(s r / 2) \partial_{\nu} f(x+s(y-x)+\operatorname{tr\nu } / 2) .
$$

But $x+s(y-x)+\operatorname{tr\nu } / 2 \in B(y, r)$; so

$$
\left(f\left(x_{2}\right)-f\left(x_{1}\right)\right) /(s r / 2) \geqq 0 .
$$

Now since $f$ is differentiable at $x$, 


$$
\begin{aligned}
f\left(x_{2}\right)-f\left(x_{1}\right)= & f(x)+(s(y-x)+\operatorname{sr\nu } / 2) \cdot \operatorname{grad} f(x) \\
& +o(|s(y-x)+\operatorname{sr\nu } / 2|) \\
& -f(x)-s(y-x) \cdot \operatorname{grad} f(x)-o(|s(y-x)|) \\
= & (\operatorname{sr\nu } / 2 \cdot \operatorname{grad} f(x)+o(|s(y-x)+\operatorname{sr\nu } / 2|) \\
& -o(|s(y-x)|) \\
= & (\operatorname{sr} / 2) \partial_{\nu} f(x)+o(s) \text { as } s \rightarrow 0
\end{aligned}
$$

(since $y, x, r$, and $\nu$ are fixed the two " $o$ " terms are just $o(s)$ ). But $\left(f\left(x_{2}\right)-f\left(x_{1}\right)\right) /(s r / 2) \geqq 0$. Thus $\partial_{\nu} f(x)+o(1) \geqq 0$ as $s \rightarrow 0$, and hence $\partial_{\nu} f(x) \geqq 0$.

That $E_{\nu, 0}$ is a $G_{\delta}$ set follows since $f$ is continuous and hence $\partial_{\nu} f$ is of Baire class one. Hence $E_{\nu, 0}$ is a ball closed $G_{\delta}$ set.

The converse is far from true. There are functions $f$ having directional derivatives in every direction and such that for each $\nu$ and for each real number $a, E_{\nu, a}$ and $E_{\nu}^{a}$ are ball closed $G_{\delta}$ sets, but $f$ is not differentiable. The situation is even worse. It is not hard to see that if $f$ is differentiable, then $E_{\nu, a}$ and $E_{\nu}^{a}$ have a much stronger closure property than just being ball closed $G_{\dot{\delta}}$ sets. Suppose that some $x$ in $E_{n}$ is the vertex of a "cone" in $E_{n}$ whose interior lies in $E_{\nu, a}\left(\operatorname{resp} . E_{\nu}^{a}\right)$. Then $x \in E_{\nu, a}$ (resp. $E_{\nu}^{a}$ ). This property on the sets $E_{\nu, a}$ and $E_{\nu}^{a}$ is still not enough to guarantee that $f$ is differentiable. For example, it is possible to construct a function $f(x, y)$ which is zero except for points $(x, y)$ satisfying $y>0$ and $y^{2}<x<4 y^{2}$, has continuous partial derivatives except at $(0,0)$ where all directional derivatives exist, however, and are zero, but $f$ is not differentiable at $(0,0)$ (If $h(t)$ is infinitely differentiable, with support $[0,1]$, then $f(x, y)=x h\left(\left(x-y^{2}\right) / 3 y^{2}\right)$ for $y>0$ and $f(x, y)=0$ for $y \leqq 0$ is such a function.) If $(x, y)$ is the vertex of a "cone" whose interior lies in $E_{\nu, a}$ (resp. $\left.E_{\nu}^{a}\right)$ and if $(x, y) \neq(0,0)$, then $\partial_{\nu} f$ is continuous at $(x, y)$; so $x \in E_{\nu, a}$ (resp. $\left.x \in E_{\nu}^{a}\right)$. If $(0,0)$ is the vertex of such a "cone," then that cone does not lie in the support of $f$; so there is an $(x, y)$ in that cone with $\partial_{\nu} f(x, y)=0$. Thus $0 \geqq a$ (resp. $\left.0 \leqq a\right)$. Since $\partial_{\nu} f(0,0)=0$, it follows that $(0,0) \in E_{\nu, a}$ (resp. $\left.(0,0) \in E_{\nu}^{a}\right)$. Consequently the sets $E_{\nu, a}$ and $E_{\nu}^{\alpha}$ contain the vertex of any cone whose interior they contain, but by choice $f$ is not everywhere differentiable.

\section{REFERENCES}

1. A. M. Bruckner and J. G. Ceder, Darboux continuity, Jber, Deutsch. Math-Verein. 67 (1965), 93-117.

2. A. Denjoy, Sur une propriété des fonctions dérivées, Enseignement Math. 18 (1916), 320-328. 
3. Michael J. Evans, $L_{p}$ derivatives and approximate Peano derivatives, Trans. Amer. Math. Soc., 165 (1972), 381-388.

4. C. Goffman and C. J. Neugebauer, On approximate derivatives, Proc. Amer. Math. Soc., 11 (1960), 962-966.

5. L. Mišik, Über die Funktionen der ersten Baireschen Klasse mit der Eigenschaft von Darboux, Mat. Fyz. Casopis Sloven. Akad. Vied, 14 (1964), 44-49.

6. C. J. Neugebauer, Darboux functions of Baire class one and derivatives, Proc. Amer. Math. Soc., 13 (1962) 838-843.

7. H. W. Olvier, The exact Peano derivative, Trans. Amer. Math. Soc., 76 (1954), 444-456.

8. C. E. Weil, On properties of derivatives, Trans. Amer. Math. Soc., 114 (1965), 363376.

9. - On approximate and Peano derivatives, Proc. Amer. Math. Soc., 20 (1969), 487-490.

10. Z. Zahorski, Sur la prémière dérivée, Trans. Amer. Math. Soc., 69 (1950), 1-54.

Received August 10, 1971. Research supported by the National Science Foundation grant GP-12293

Michigan State UNIVERSITY 



\section{PACIFIC JOURNAL OF MATHEMATICS}

\section{EDITORS}

\section{H. SAMELSON}

Stanford University

Stanford, California 94305

C. R. Новву

University of Washington Seattle, Washington 98105

\section{J. DuGundJI}

Department of Mathematics University of Southern California Los Angeles, California 90007

RICHARD ARENS

University of California Los Angeles, California 90024

\section{ASSOCIATE EDITORS}
E. F. BECKENBACH
B. H. NeumanN
F. WOLF
K. YoSHIDA

\section{SUPPORTING INSTITUTIONS}

\author{
UNIVERSITY OF BRITISH COLUMBIA \\ CALIFORNIA INSTITUTE OF TECHNOLOGY \\ UNIVERSITY OF CALIFORNIA \\ MONTANA STATE UNIVERSITY \\ UNIVERSITY OF NEVADA \\ NEW MEXICO STATE UNIVERSITY \\ OREGON STATE UNIVERSITY \\ UNIVERSITY OF OREGON \\ OSAKA UNIVERSITY
}

\author{
UNIVERSITY OF SOUTHERN CALIFORNIA \\ STANFORD UNIVERSITY \\ UNIVERSITY OF TOKYO \\ UNIVERSITY OF UTAH \\ WASHINGTON STATE UNIVERSITY \\ UNIVERSITY OF WASHINGTON \\ $*{ }^{*}$
AMERICAN MATHEMATICAL SOCIETY
NAVAL WEAPONS CENTER
}

The Supporting Institutions listed above contribute to the cost of publication of this Journal, but they are not owners or publishers and have no responsibility for its content or policies.

Mathematical papers intended for publication in the Pacific Journal of Mathematics should be in typed form or offset-reproduced, (not dittoed), double spaced with large margins. Underline Greek letters in red, German in green, and script in blue. The first paragraph or two must be capable of being used separately as a synopsis of the entire paper. The editorial "we" must not be used in the synopsis, and items of the bibliography should not be cited there unless absolutely necessary, in which case they must be identified by author and Journal, rather than by item number. Manuscripts, in duplicate if possible, may be sent to any one of the four editors. Please classify according to the scheme of Math. Rev. Index to Vol, 39. All other communications to the editors should be addressed to the managing editor, Richard Arens, University of California, Los Angeles, California, 90024.

50 reprints are provided free for each article; additional copies may be obtained at cost in multiples of 50 .

The Pacific Journal of Mathematics is issued monthly as of January 1966. Regular subscription rate: $\$ 48.00$ a year (6 Vols., 12 issues). Special rate: $\$ 24.00$ a year to individual members of supporting institutions.

Subscriptions, orders for back numbers, and changes of address should be sent to Pacific Journal of Mathematics, 103 Highland Boulevard, Berkeley, California, 94708.

PUBLISHED BY PACIFIC JOURNAL OF MATHEMATICS, A NON-PROFIT CORPORATION

Printed at Kokusai Bunken Insatsusha (International Academic Printing Co., Ltd.), 270, 3-chome Totsuka-cho, Shinjuku-ku, Tokyo 160, Japan. 


\section{Pacific Journal of Mathematics}

\section{Vol. 44, No. $2 \quad$ June, 1973}

Tsuyoshi Andô, Closed range theorems for convex sets and linear liftings . . . . . . 393

Richard David Bourgin, Conically bounded sets in Banach spaces . . . . . . . . . 411

Robert Jay Buck, Hausdorff dimensions for compact sets in $R^{n} \ldots \ldots \ldots \ldots \ldots \ldots . \ldots 421$

Henry Cheng, A constructive Riemann mapping theorem ................ 435

David Fleming Dawson, Summability of subsequences and stretchings of

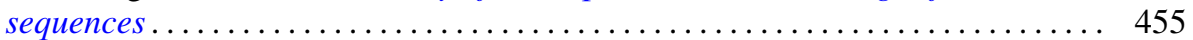

William Thomas Eaton, A two sided approximation theorem for 2-spheres ....... 461

Jay Paul Fillmore and John Herman Scheuneman, Fundamental groups of compact complete locally affine complex surfaces ....................... 487

Avner Friedman, Bounded entire solutions of elliptic equations . . . . . . . . . . . 497

Ronald Francis Gariepy, Multiplicity and the area of an $(n-1)$ continuous

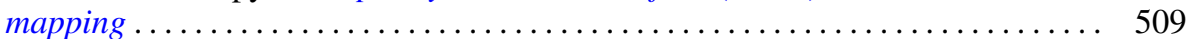

Andrew M. W. Glass, Archimedean extensions of directed interpolation groups . . . . 515

Morisuke Hasumi, Extreme points and unicity of extremum problems in $H^{1}$ on

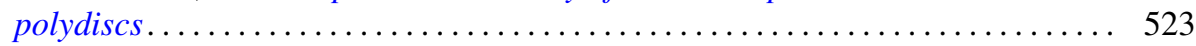

Trevor Ongley Hawkes, On the Fitting length of a soluble linear group . . . . . . 537

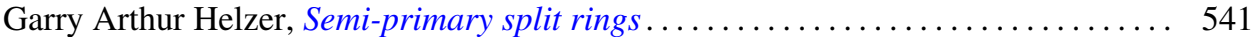

Melvin Hochster, Expanded radical ideals and semiregular ideals . . . . . . . . . 553

Keizō Kikuchi, Starlike and convex mappings in several complex variables . . . . . . 569

Charles Philip Lanski, On the relationship of a ring and the subring generated by its

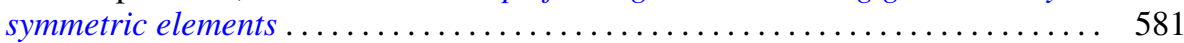

Jimmie Don Lawson, Intrinsic topologies in topological lattices and semilattices ........................................... 593

Roy Bruce Levow, Counterexamples to conjectures of Ryser and de Oliveira ...... 603

Arthur Larry Lieberman, Some representations of the automorphism group of an infinite continuous homogeneous measure algebra ..........

William George McArthur, $G_{\delta}$-diagonals and metrization theorems $\ldots .$.

James Murdoch McPherson, Wild arcs in three-space. II. An invariant of

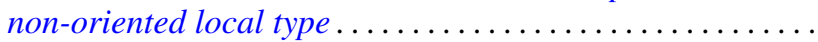

H. Millington and Maurice Sion, Inverse systems of group-valued measures ...

C. Edward Moore, Concrete semispaces and lexicographic separation of convex

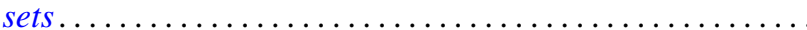

Jingyal Pak, Actions of torus $T^{n}$ on $(n+1)$-manifolds $M^{n+1}$.

Merrell Lee Patrick, Extensions of inequalities of the Laguerre and Turán type . . . . 675

Harold L. Peterson, Jr., Discontinuous characters and subgroups of finite index. . . . 683

S. P. Philipp, Abel summability of conjugate integrals . . . . . . . . . . . . . 693

R. B. Quintana and Charles R. B. Wright, On groups of exponent four satisfying an

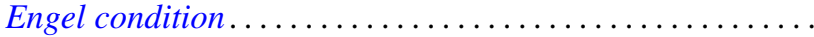

Marlon C. Rayburn, On Hausdorff compactifications. . . . . . . . . .

Martin G. Ribe, Necessary convexity conditions for the Hahn-Banach theorem in

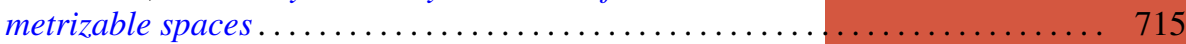

Ryōtarō Satō, On decomposition of transformations in infinite measure spaces .... 733

Peter Drummond Taylor, Subgradients of a convex function obtained from a

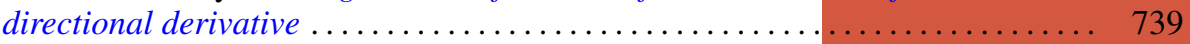

James William Thomas, A bifurcation theorem for $k$-set contractions . . . . . . . . 749 Clifford Edward Weil, A topological lemma and applications to real functions . . . . 757

Stephen Andrew Williams, A nonlinear elliptic boundary value problem . . ....... 767

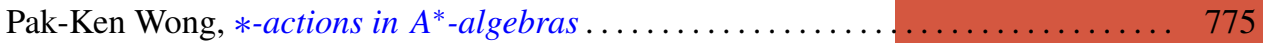

